\title{
A proposal of information system architecture for public transport
}

\author{
C. García, G. Padrón, F. Alayón \& J. Caraballo \\ Department of Computer Science and Systems, \\ Las Palmas de Gran Canaria University, Spain
}

\begin{abstract}
In order to provide a quality service to the clients, the public road transport companies have to perform operations scheduling. To achieve this goal, these companies have to handle various types of information: geographic, traffic, statistical, technical, etc. Processing these kinds of information leads to a problem: the high level of dynamism and high volume of data implied. In this paper we explain a system model to resolve this problem; this model is based on distributed computing and remote sensing. Nowadays, this model is used in the metropolitan road transport of Gran Canaria (Canary Islands, Spain) and it enables the automatic processing of all the information related to the production, generated by a fleet of 305 buses, transporting more than 30,000,000 passengers and controlling all the planning operations of the fleet.
\end{abstract}

Keywords: management information system, intelligent transport systems, geographic information system and remote sensing.

\section{Introduction}

An important objective of the transport companies is to achieve a high level of fulfilment of service planning. This is an important requirement to provide the clients with a quality service and to guarantee the efficiency of the vehicle fleet operations. To achieve this goal, it is necessary to handle proper information. It implies to handling information obtained at the time (real time requirement) and in the required amount. This information represents the activity of the company, the resources used (drivers and vehicles), the precise time and the geographical area. The transport company has to evaluate the level of fulfilment of the plan, in order to provide information to the client, to act in response to incidents which 
affect the service and to improve the operations scheduling. To make this evaluation it is first necessary to identify the factors that affect the production operations. Second, we have to obtain the data representations of these factors, and third, we need to process, in the correct way, these data in order to obtain all the information needed for controlling, reporting and planning the production activity of the fleet. In this paper we describe the architecture of an integral information system developed by us that facilitates the identification of the production factors and permits the controlling, reporting and planning in a successful way.

\section{Required information identification}

Depending on the level of automation of the production tasks of the public road transport company, different amounts and types of data are required. In order to automate these tasks we first need to identify the required information for each element of the production. The second step is to know the timing requirements of the information process, and finally, we have to calculate the amounts of data required. Following this scheme, we find different data components associated to different levels of automation of the production tasks:

- Basic data components: they consist of the necessary elements to achieve a minimum level of automation of the production of the transport company; basically these data are associated with the payment of the clients and the drivers taking.

- Intermediate data components: they consist of the necessary elements to achieve the control operation of the resources (drivers and vehicles); basically, these data elements are associated with the control of scheduled tasks.

- Advance data components: they consist of the necessary elements to achieve advanced functionalities, such as real time information to the clients, optimum response to events produced during the production activities and the improvement of the operations planning.

To build each component, we need to handle two kinds of supplementary information: geographical and information about passengers flows. We can represent these information requirements as a layered structure configured by three horizontal data components and two vertical data components.

\section{Goals and requirements}

The main objective of the system is to automate all the data flow related to the production activity of the transport corporation. The achievement of this goal implies the automatic execution of all the tasks related to the production activity: payments, drivers' takings, operation control, service information to the clients and planning. This automatic flow is reached by the acquisition processes executed on board systems (mobile systems), statistical and validation processes executed on the corporation hosts and finally, the feedback processes to update 
all the information handled by the platform integrated in the system. The final objective is to automatically execute these three steps of this cyclic flow of information. From a functional point of view, to reach this high level of automation, we have the following requirements:

- Mobile information system with the capacity to acquire, storage and process all the relevant data, necessary to represent the vehicles activities.

- Acquiring the data at the proper time.

- Controlling in order to act in case of production incidents.

- Tools that permit evaluation of the level of fulfilment achieved by the vehicles operation and to build new data representations of the production plan.

- Tools that permit to feedback new data representations, used by the mobile platforms and other special stations of the information system, such as information kiosks for clients.

Additionally, other requirements related to the effective cost of the resources used in the production activities must be considered; specifically, we are concerned with the communications infrastructure and the maintenance of the mobile information systems. With regards to the communication infrastructure the critical parameters are: the band width, the connection establishment time and the scheme used to monitor the state of the fleet (polling versus exception model). With regards to the maintenance of the mobile information systems fleet, it is critical to have tools that allow preventive maintenance and administration of the on board elements in an unsupervised and remote way.

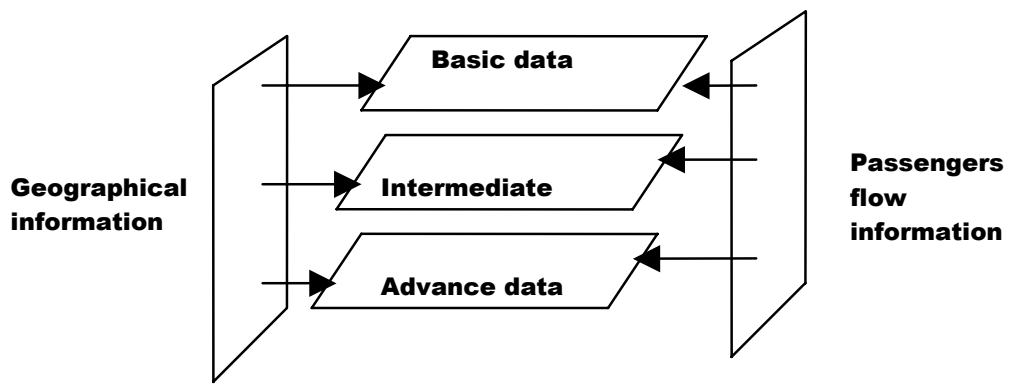

Figure 1: General vision of the data organization.

\section{System architecture}

The architecture of the information system is configured by several modules and these modules are structured in two subsystems: the production management information subsystem and the communication services subsystem. The 
production management information subsystem is configured by the following modules:

- Mobile information platforms: they are responsible for registering, monitoring, controlling and communicating all the operations executed on the vehicles. The mobile platforms are equipped with all the necessary devices to perform these tasks [1]. Specifically, they have elements of sensing to control the on board physical conditions (power, temperature), to register the combustible consumption and to record scene images for safety purposes. Another important element of the on board platform is the GPS receiver used to control the vehicles geographic location and to have a common reference timer in order to synchronise all fleet operations. The model used to control the operation of the vehicles is another relevant aspect. The mobile platforms are controlled by an exception scheme, which implies that communication with the control node of the corporative network is only established when a relevant event is produced. This control scheme optimizes the cost of communications.

- Fleet control module: it controls the fleet operations and it can act to guarantee the fulfilment of the plan. There are various types of events that can affect the plan: technical alarms, time non-fulfilments, non-planning operation, etc. All these events are represented using different types of information (time, location, vehicles, driver, etc) and stored in on board log files and also in the corporative data base.

- Takings module: this module is responsible of the on board payments. It provides information about the taking associated to different elements of the production (drivers, vehicles, lines, routes, etc).

- Client information module: the objective of this element is to provide information about the company services to the client in real time. This information is originated from planning and it is modified by the exceptions received from mobile platforms.

- Planning subsystem module: this element produces the operations plan for the fleet, specifying the resources (drivers and vehicles) required and the time for each operation. The main goal of this subsystem is to respond to the clients demand optimising the resources used.

- Maintenance module: this element supports all the tasks related to the maintenance and administration of all remote special platforms integrated in the corporative information system. These platforms are the on board mobile system and the information point for clients. The goals of this module are: to facilitate maintenance, to improve the response time to technical alarms and to optimize the maintenance costs. These goals are reached by the automatic execution of all the maintenance tasks.

All the communications between the previous modules are executed by the communication services subsystem. This subsystem is configured by two modules, structured in a layered way. These modules are:

- Network module: it is responsible for integrating the mobile platform in the corporative network. Nowadays, to achieve this integration, this element 
uses three kinds of communication infrastructures: a radio system to transmit data packets associated to exceptions generated in the fleet operations, a wireless local network to transmit a large volume of data to the mobile platform and on board ad-hoc network to implement a payment system based on contactless intelligent cards.

- Transactions module: This module achieves the automatic data flow with the mobile platform, executing all the required data validation.

All the processes of the production management information subsystem can be classified in the following way:

- Acquisition: they are responsible of acquiring any data that represents a relevant aspect of the production environment; physical (positioning and sensing) and logical (states of the vehicles, clients demand, etc). All these processes belong to the mobile information platforms.

- Monitoring: these processes are responsible of verifying and measuring the level of fulfilment of the plan. They are able to detect events that can affect the plan, and to transmit them to the control module. These processes are executed on the mobile information platforms, and their design was influenced by administration networks [2] and pervasive computing models [3], in order to fulfil a high level of scalability, interoperability and flexibility.

- Control: the goal of these processes is to provide solutions to operation events such as: technical alarms, planning non-fulfilments and events non-planning. From the point of view of the transport corporation, the objective is to establish uniform criterions and actuations to resolve the production events in order to guarantee the suitability of the exceptions solutions.

- Optimization: this category is formed by all the processes that can modify the plan in order to improve it. To reach this improvement, these processes acquire information from the control module, in order to obtain systematic exceptions of the current plan, from the statistical module, to obtain relevant parameters about the clients demand and from the mobile information module, to obtain physical parameters of the production environment such as: routes times, geographical representations of the routes, vehicles velocity, etc.

- Statistical: this category is configured by all the processes that can obtain relevant parameters by applying statistical methods. These parameters are of different types. For example: time of routes, takings associated to different production elements, origin-destination client demands, levels of occupation of the vehicles, etc.

- Visualization: all the processes that present information to the system user in a friendly way belong to this process category. The main requirement that this kind of processes have to satisfy is to present the information independently of the type of device used by the user (cellular phone, PDAs, driver console, desktop computer, etc). For this reason, the accessibility techniques play a relevant role in these processes [4]. 
A critical aspect of the system architecture is the data management, because of the high volume and complexity of data. For this reason, the data base used is a critical resource of the system. This data base is configured by four layered components. These are:

- Level 0: geographic data base where all the geographic entities of the production are represented, such as: geographical points, geographical representations of roads, etc.

- Level 1: data base of the transport network where the entities of the geographic data base are combined to build the elements of the transport network, incorporating entities such us: route time, bus stops, lines, expeditions, time table, etc.

- Level 2: planning exceptions data base where all the productions incidents are represented, using attributes such as: exception type, geographical positions, time, vehicles, etc

- Level 3: planning data base where all the operation of the production are planned, specifying the following attributes: operation type, time when the operation must be started or finished, place where the operation has to be executed, etc.

- Level 4: production results data base where all the results of the production are represented. These results are associated to on board payments and clients demands.

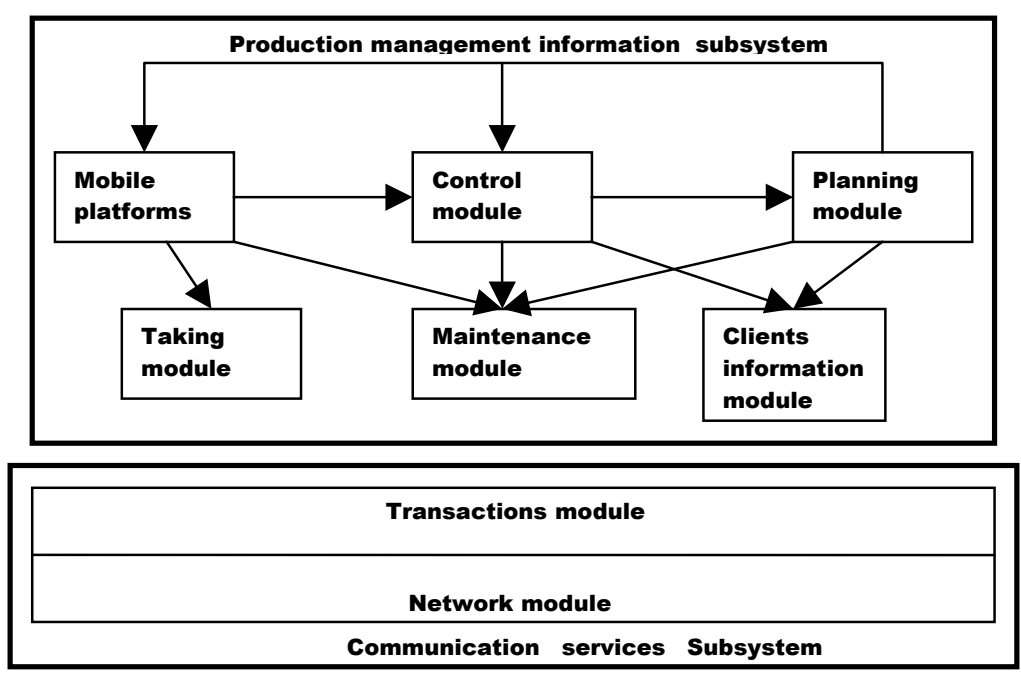

Figure 2: $\quad$ General vision of the system architecture. 


\section{Practical results}

Nowadays, this system used in the public road transport company Global SalcaiUtinsa S.A. It operates in Gran Canaria (Canary Islands, Spain) with a fleet of 305 buses, executing about 1,000,000 expeditions and transporting about $30,000,000$ passengers yearly. All the information required and produced in the production of this company is processed by the system. In order to have an idea about the volume and complexity of this information, we present some examples.

The information about the network transport represents more than 2700 bus stops that are used in 770 different lines. The system controls the timetable fulfilment of each expedition: when a new expedition starts, the timetable fulfilment is achieved using a set of control points which are represented by the GPS position (longitude, latitude and altitude), the arriving time and the time thresholds to generate the alarms of delayed and early arrivals. The time thresholds can be define in a dynamic way depending on the lines, times of the expeditions or the control points.

The information about the plan represents the operation of 700 drivers and 305 vehicles. These operations are grouped in more than 2000 working days that contain more than 4000 expeditions a day. All the working days are monitored by the mobile platforms and controlled by the control station in real time. The corporation knows the position of the basic resources (buses and drivers) because when each driver start his job, the on board system generates a warning to the control station informing the bus used, the geographical position, the time and the driver who starts the service. Any exception to the plan is communicated to the control station by an alarm data packet that contains all the information needed to resolve exception (time, location, bus, driver, line, etc), each alarm packet received is registered in the exceptions data base adding the arrived time of the packet. With this information the corporation can evaluate the communications infrastructure performance and the exception response time.

The results of the production are processed in real time. As a consequence, the company can achieve the on board payment takings in an unsupervised and distributed way, using automatic cashiers. Another capability achieved consists of reporting the production results in real time.

The maintenance of all the special platforms of the information system (on board platform and information kiosks for clients) is managed in an unsupervised and remote way. The figure 3 describes the percentages of buses and the elapsed days to achieve software updates in all mobile information systems of the buses fleet.

\section{Conclusions}

In this paper we have presented a information system architecture for public transport which permits a high level of automation in all the process related to the production activity of the transport corporation. Specifically, tasks that require a high amount of data and short response time. A relevant aspect of the architecture is the management of various communications infrastructures that 

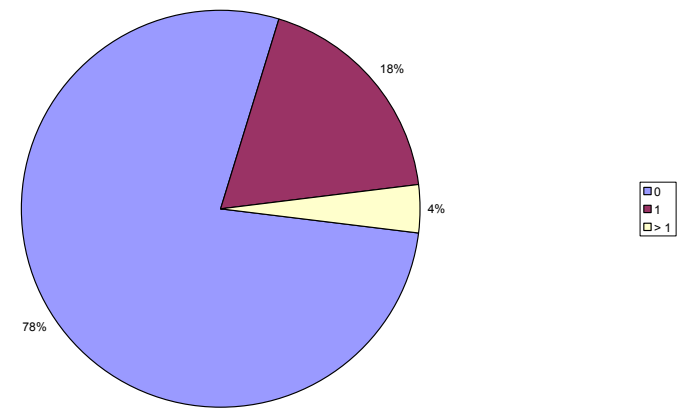

Figure 3: $\quad$ Buses percentages and elapsed days to achieve software updates.

integrate in a proper way the mobile information platforms of the vehicles in the corporation information system. This proper integration is achieved using the communications infrastructures in an efficient way, from the technical and effective cost points of view. To fulfil this requirement we have applied models and techniques based on pervasive paradigms. From the point of view of the information, the architecture models all the required data in a layered and hierarchical way, this model integrates data sets which represents different relevant aspects of the transport corporation: geographical, technical needed to maintenance, production results, etc. This data model permits us to organize the required high amount of data in order to identify the data requirements, the relationships between them and to automate the data maintenance. Finally, we report that this proposal system architecture is working in Gran Canaria (Canary Islands) to manage the production activity of the public metropolitan transport, controlling the activity of a fleet of 300 buses which execute about 1,000,00 expeditions transporting about $30,000,00$ passengers yearly.

\section{References}

[1] Garcia, C., Alayón, F., Medina, P. \& Fernández, E., On board system model for road intelligent transport, Urban Transport VIII, series: Advances in Transport, vol 12. , pp. 213-220, WIT Press, 2002.

[2] Stallings, W., SNMP, SNMPv2, SNMPv3, and RMON1, Addison_Wesley, 1999.

[3] Saha, D. \& Mukherjee, A., Pervasive Computing. A paradigm for $21^{\text {st }}$ Century, Computer, vol. 36, n 3, 2003.

[4] Loiacono, E., Improving Web Accessibility. Computer, Vol. 36, $\mathrm{N}^{\mathrm{o}} 1$, pp 117-119, 2003. 